INHA-NTG-01/2013

\title{
Transverse charge densities in the nucleon in nuclear matter
}

\author{
Ulugbek Yakhshiev* and Hyun-Chul Kim $\dagger^{\circledast}$ \\ Department of Physics, Inha University, Incheon 402-751, Republic of Korea
}

(Dated: April 2013)

\begin{abstract}
We investigated the transverse charge densities in the nucleon in nuclear matter within the framework of the in-medium modified Skyrme model. The medium modification of the nucleon electromagnetic form factors are first discussed. The results show that the form factors in nuclear matter fall off faster than those in free space, as the momentum transfer increases. As a result, the charge radii of the nucleon become larger, as the nuclear matter density increases. The transverse charge densities in the nucleon indicate that the size of the nucleon tends to bulge out in nuclear matter. This salient feature of the swelling is more clearly observed in the neutron case. When the proton is transversely polarized, the transverse charge densities exhibit the distortion due to the effects of the magnetization.
\end{abstract}

PACS numbers: 12.39.Dc, 13.40.Gp, 14.20.Dh

Keywords: Skyrmions, Electromagnetic form factors, Protons and neutrons.

1. It is of utmost importance to understand the structure of the nucleon in particle and nuclear physics, since the nucleon consists of the basic building block of matter. In particular, the nucleon electromagnetic (EM) form factors are the fundamental issue in that they reveal how the electric charge and magnetization of the quarks are distributed inside the nucleon. While the EM form factors have been studied well over several decades, their understanding is still not complete.

Recently, a series of new measurements of the EM form factors has been carried out and has produced the remarkably precise data $[1] 8$. These new experimental results have subsequently intrigued both experimental and theoretical works (see, for example, the following reviews [9 11]). The experimental data with high precision enable one to make a flavor decomposition of the nucleon EM form factors with isospin and charge symmetries taken into account [12, 13. Moreover, generalized parton distributions (GPDs) have unveiled a novel aspect of the nucleon EM structure: The Fourier transforms of the nucleon EM form factors in the transverse plane, as viewed from a light front frame moving towards a nucleon, paint a tomographic picture of how the charge densities of quarks are distributed [14, 15] transversely. These transverse charge densities of the quarks inside a nucleon have been already investigated for the unpolarized [16] and transversely polarized [17] nucleons.

It is of equal importance to examine how the EM structure of the nucleon is changed in nuclear matter. Studying the EM form factors of the nucleon in medium provides a new aspect on EM properties of the nucleon modified in nuclei. In fact, the first experimental study of deeply virtual Compton scattering on (gaseous) nuclear targets $(\mathrm{H}, \mathrm{He}, \mathrm{N}, \mathrm{Ne}, \mathrm{Kr}, \mathrm{Xe})$ was reported in 18 . While uncertainties of the first measurement are so large that one is not able to observe nuclear modifications

\footnotetext{
* yakhshiev@inha.ac.kr

$\dagger$ hchkim@inha.ac.kr
}

of the nucleon structure, Future experiments will bring about more information on medium modifications of the EM properties of the nucleon.

In the present work, we want to investigate the nucleon EM form factors and the transverse charge densities of quarks inside a nucleon in nuclear matter within the framework of an in-medium modified Skyrme model. The Skyrme model has certain virtues: it is simple but respects chiral symmetry and its spontaneous symmetry breaking. Moreover, one can easily extend it to the study of nuclear matter, based on modifications of the pion in medium [19, 20. The energy-momentum tensor form factors of the nucleon, which are yet another fundamental form factors that are related to the generalized EM form factors, have been investigated in nuclear matter within this framework 21. The results have explained certain interesting features of the modifications of the nucleon in nuclear matter such as the pressure and angular momentum. Indeed, we will also show in this work how the EM properties of the nucleon are changed in nuclear matter in a simple manner. We will also see that the transverse charge densities expose noticeably how the distribution of quarks undergo changes in the presence of nuclear medium.

2. We begin with the in-medium modified effective chiral Lagrangian [20]:

$$
\begin{aligned}
\mathcal{L}^{*}= & \frac{F_{\pi}^{2}}{16} \operatorname{Tr}\left(\frac{\partial U}{\partial t}\right)\left(\frac{\partial U^{\dagger}}{\partial t}\right) \\
& -\frac{F_{\pi}^{2}}{16} \alpha_{p}(\boldsymbol{r}) \operatorname{Tr}(\boldsymbol{\nabla} U) \cdot\left(\boldsymbol{\nabla} U^{\dagger}\right) \\
& +\frac{1}{32 e^{2} \gamma(\boldsymbol{r})} \operatorname{Tr}\left[U^{\dagger} \partial_{\mu} U, U^{\dagger} \partial_{\nu} U\right]^{2} \\
& +\frac{F_{\pi}^{2} m_{\pi}^{2}}{16} \alpha_{s}(\boldsymbol{r}) \operatorname{Tr}\left(U+U^{\dagger}-2\right),
\end{aligned}
$$

where $F_{\pi}=108.78 \mathrm{MeV}$ denotes the pion decay constant, $e=4.85$ the Skyrme parameter, and $m_{\pi}$ the experimental value of the pion mass $m_{\pi^{0}}=134.98 \mathrm{MeV}$. This set of the parameters reproduce qualitatively well the exper- 
imental data for the nucleon and $\Delta$-isobar in free space.

The medium functionals, $\alpha_{s}, \alpha_{p}$ and $\gamma$, are expressed as

$$
\begin{aligned}
\alpha_{s} & =1-\frac{4 \pi b_{0} \rho(\boldsymbol{r}) f}{m_{\pi}^{2}} \\
\alpha_{p} & =1-\frac{4 \pi c_{0} \rho(\boldsymbol{r})}{f+g_{0}^{\prime} 4 \pi c_{0} \rho(\boldsymbol{r})}, \\
\gamma & =\exp \left(-\frac{\gamma_{\mathrm{num}} \rho(\boldsymbol{r})}{1+\gamma_{\operatorname{den}} \rho(\boldsymbol{r})}\right) .
\end{aligned}
$$

They encode information on how the surrounding environment influences properties of the single skyrmion. The parameters $\alpha_{s}$ and $\alpha_{p}$ are related to the corresponding phenomenological $S$ - and $P$-wave pion-nucleus scattering lengths and volumes, i.e. $b_{0}=-0.024 m_{\pi}^{-1}$ and $c_{0}=0.06 m_{\pi}^{-3}$, respectively, which describe the pion physics in a nucleus [22]. The last functional $\gamma$ is parameterized in the form of an exponential function and represents the medium modification of the Skyrme parameter. This simple form with two variational parameters $\gamma_{\text {num }}=0.47 m_{\pi}^{-3}$ and $\gamma_{\text {den }}=0.17 m_{\pi}^{-3}$ reproduce the correct position of the saturation point of symmetric nuclear matter [20]. The $\rho$ stands for the density of nuclear matter. The $g_{0}^{\prime}=0.7$ denotes the Lorentz-Lorenz or correlation parameter and $f=1+m_{\pi} / m_{N}^{\text {free }}$ is the kinematical factor.

Since we consider isospin symmetric infinite nuclear matter in the present work, the density can be regarded as a homogeneous and constant one. Thus, we can simply choose the spherically symmetric "hedgehog" for the soliton:

$$
U=\exp \{i \boldsymbol{n} \cdot \boldsymbol{\tau} F(r)\}
$$

where $\boldsymbol{n}$ denotes the unit radial vector in coordinate space and $\boldsymbol{\tau}$ the usual Pauli isospin matrices.

3. We refer to Ref. [20] for the details of the minimization procedure and other useful formulas. In this work, we concentrate on EM properties of the nucleon.

The nucleon matrix element of the EM current is expressed in terms of the Dirac and Pauli form factors:

$$
\begin{aligned}
& \left\langle N\left(p^{\prime}, S^{\prime}\right)\left|J_{\mu}^{E M}(0)\right| N(p, S)\right\rangle \\
& =\bar{u}_{N}\left(p^{\prime}, S^{\prime}\right)\left[\gamma_{\mu} F_{1}^{*}\left(q^{2}\right)+i \frac{\sigma_{\mu \nu} q^{\nu}}{2 m_{N}} F_{2}^{*}\left(q^{2}\right)\right] u_{N}(p, S),(4)
\end{aligned}
$$

where the EM current is defined in terms of the baryon current $B_{\mu}$ and the isovector current $J_{\mu}^{(3)}$

$$
J_{\mu}^{E M}(0)=\frac{1}{2}\left(B_{\mu}(0)+J_{\mu}^{(3)}(0)\right) .
$$

The $\gamma_{\mu}$ denotes the Dirac matrices and $u_{N}(p, S)$ stands for the Dirac spinor for the nucleon with mass $m_{N}$, momentum $p$, and the third component of its spin $S$. The $\sigma_{\mu \nu}$ is the spin operator $i\left[\gamma_{\mu}, \gamma_{\nu}\right] / 2$ and $q^{2}$ the square of the momentum transfer $q^{2}=-Q^{2}$ with $Q^{2}>0$. The asterisk means the form factors in medium. In the Breit frame, the in-medium modified Sachs EM form factors of the nucleon are defined as

$$
\begin{aligned}
G_{E}^{*}\left(Q^{2}\right) & =\frac{1}{2} \int \mathrm{d}^{3} r e^{i \boldsymbol{q} \cdot \boldsymbol{r}} J_{0}^{E M}(\boldsymbol{r}) \\
G_{M}^{*}\left(Q^{2}\right) & =\frac{m_{N}}{2} \int \mathrm{d}^{3} r e^{i \boldsymbol{q} \cdot \boldsymbol{r}}\left[\boldsymbol{r} \times \boldsymbol{J}^{E M}(\boldsymbol{r})\right],
\end{aligned}
$$

where $J^{0}$ and $\boldsymbol{J}$ correspond respectively to the temporal and spatial components of the properly normalized sum of the baryonic (topological) current $B_{\mu}$ and the third component of the isovector (Noether) current $V_{\mu}^{*}$.

The isoscalar $(S)$ and isovector EM formfactors are generically expressed as

$$
G_{E, M}^{S, V, *}\left(Q^{2}\right)=\int \mathrm{d}^{3} r e^{i q r \cos \theta} \rho_{E, M}^{S, V}(r, \theta),
$$

where the densities are given as

$$
\begin{aligned}
\rho_{\mathrm{E}}^{\mathrm{S}}(r, \theta)= & -\frac{F^{\prime} \sin ^{2} F}{4 \pi^{2} r^{2}}, \\
\rho_{\mathrm{M}}^{\mathrm{S}}(r, \theta)= & -\frac{m_{N}}{8 \pi^{2} \lambda^{*}} F^{\prime} \sin ^{2} F \sin ^{2} \theta, \\
\rho_{\mathrm{E}}^{\mathrm{V}}(r, \theta)= & \frac{\sin ^{2} F}{12 \lambda^{*}}\left\{F_{\pi}^{2}+\frac{4}{e^{2} \gamma}\left(F_{r}^{2}+\frac{\sin ^{2} F}{r^{2}}\right)\right\}, \\
\rho_{\mathrm{M}}^{\mathrm{V}}(r, \theta)= & \frac{m_{N}}{3}\left\{F_{\pi}^{2} \alpha_{p}+\frac{4}{e^{2} \gamma}\left(F^{\prime 2}+\frac{\sin ^{2} F}{r^{2}}\right)\right\} \\
& \times \sin ^{2} F \sin ^{2} \theta .
\end{aligned}
$$

Here, $\lambda^{*}$ stands for the medium-modified moment of inertia of the soliton. Note that because of the modification of the Skyrme term, all of the electromagnetic charge distributions except for the isoscalar electric charge depend explicitly on the medium density. Moreover, the medium functional $\alpha_{p}$ appears in the expression of the isovector magnetic density distribution.

The charges of the proton $(p)$ and neutron $(n)$ are defined as

$$
\left(\begin{array}{c}
Q^{p} \\
Q^{n}
\end{array}\right):=\int \mathrm{d}^{3} r\left\{\rho_{E}^{S}(r, \theta) \pm \rho_{E}^{V}(r, \theta)\right\},
$$

where density distributions include the prefactor $1 / 2$. Similarly, the magnetic moments of the nucleon are defined as

$$
\left(\begin{array}{l}
\mu_{p} \\
\mu_{n}
\end{array}\right):=\int \mathrm{d}^{3} r\left\{\rho_{M}^{S}(r, \theta) \pm \rho_{M}^{V}(r, \theta)\right\} .
$$

The corresponding isoscalar and isovector mean square charge radii are given as

$$
\begin{aligned}
\left\langle r^{2}\right\rangle_{I=0}^{*}= & -\frac{2}{\pi} \int_{0}^{\infty} F^{\prime} \sin ^{2} F r^{2} \mathrm{~d} r \\
\left\langle r^{2}\right\rangle_{I=1}^{*}= & \frac{2 \pi}{3 \lambda^{*}} \int_{0}^{\infty}\left[F_{\pi}^{2}+\frac{4}{e^{2} \gamma}\left(F^{\prime 2}+\frac{\sin ^{2} F}{r^{2}}\right)\right] \\
& \times \sin ^{2} F r^{4} \mathrm{~d} r .
\end{aligned}
$$

4. We are now in a position to discuss the results. In Table I] we list the results of the magnetic moments, the 
isoscalar and isovector charge radii of the nucleon as functions of $\rho / \rho_{0}$. The electric charge radius of the nucleon is well reproduced in free space, compared with the experimental data: $(0.877 \pm 0.005) \mathrm{fm}$. The results of the proton

\begin{tabular}{ccccc}
\hline \hline$\rho / \rho_{0}$ & $\mu_{p}^{*}[\mathrm{n} . \mathrm{m}]$. & $\mu_{n}^{*}[\mathrm{n} . \mathrm{m}]$. & $\sqrt{\left\langle r_{p}^{2}\right\rangle^{*}}[\mathrm{fm}]$ & $\left\langle r_{n}^{2}\right\rangle^{*}\left[\mathrm{fm}^{2}\right]$ \\
\hline 0.0 & 1.965 & -1.238 & 0.882 & -0.316 \\
0.5 & 2.134 & -1.430 & 0.926 & -0.310 \\
1.0 & 2.315 & -1.634 & 0.966 & -0.304 \\
\hline \hline
\end{tabular}

TABLE I. Magnetic moments, meansquare radii of the nucleons in nuclear matter. Magnetic moments are given in units of nuclear magnetons (n.m.).

charge radius shows that the size of the proton swells up in nuclear matter, which is consistent with those of different approaches. For example, at normal nuclear matter density the proton charge radius $\sqrt{\left\langle r_{p}^{2}\right\rangle^{*}}$ is enhanced by about $10 \%$. On the other hand, the magnitude of the neutron charge radius $\left\langle r_{n}^{2}\right\rangle^{*}$ is reduced by about $4 \%$. At first glance, the size of the neutron seems reduced. In fact, however, it swells like that of the proton, because the positive charges of the neutron are distributed in the inner part of, whereas the negative charges are found in outer part. When the neutron is in medium, both the positive and negative charges are broadened, which implies that size of the neutron actually becomes larger in nuclear matter. This will be explicitly shown later when we discuss the transverse charge densities of the neutron.

As was known already, the results of the nucleon magnetic moments in the Skyrme model turn out to be underestimated. However, since we are mainly interested in the modification of the nucleon magnetic moments in nuclear matter, we will concentrate on how the magnetization of the nucleon undergoes changes in medium. The magnetic moments are even more dramatically changed due to the additional medium factor $\alpha_{p}$ in the expression of the magnetic-vector density distribution $\rho_{M}^{V}$. As a result, the proton magnetic moment is enhanced by almost about $18 \%$ at normal nuclear matter density $\rho_{0}$, whereas the neutron one increases by about $30 \%$. It indicates that the neutron is changed more sensitively, which will be shown later again in the neutron transverse charge densities.

Figure 1 draws the results of the proton and the neutron EM form factors in free space (dashed curve) and at normal nuclear matter density $\rho_{0}$ in nuclear matter (solid curve), respectively. We observe that as the density increases, the EM form factors fall off in general faster than those in free space. Similar qualitative results have been obtained in the framework of the quark meson coupling (QMC) model 23]. However, the present results are quantitatively different from those in the QMC model. For example, compared with the proton electric form factor at $Q^{2}=0.3 \mathrm{GeV}^{2}$ in free space, $G_{E}^{p *}\left(Q^{2}\right)$ is reduced by about $30 \%$ at $\rho_{0}$, whereas that from the QMC model decreases only by about $8 \%$ [23]. In the case of the neutron one $G_{E}^{n *}\left(Q^{2}\right)$, it is the other way around. At $Q^{2}=0.3 \mathrm{GeV}^{2}$, the neutron electric form factor is changed by about $1 \%$ only but in the QMC model brings it is lessened by about $8 \%$. However, the neutron electric form factor falls off faster in nuclear matter than that in free space (e.g. at $Q^{2}=1 \mathrm{GeV}^{2} G_{E}^{n *}$ is decreased by approximately $30 \%)$. On the other hand, the present results are similar to those of Ref. 24. In general, the neutron is more likely to be changed in medium.

The modifications of the nucleon magnetic form factors are also more prominent within the present model in comparison with the QMC model. At normal nuclear matter $\rho=\rho_{0}$ and at $Q^{2}=0.3 \mathrm{GeV}^{2}$ the proton and neutron magnetic form factors are reduced by about $12 \%$ and $8, \%$, respectively, compared to the case of the free space within our approach, while the QMC model yields them decreased by $1.5 \%$ and $0.9 \%$, respectively. Thus, in general, the present results show stronger medium effects than those from the QMC model.

It is also of great interest to study the flavordecomposed EM form factors, since the experimental data are now available in free space [12, 13. We want to examine how the flavor-decomposed EM form factors undergo the changes in nuclear matter. Assuming isospin symmetry in flavor $\mathrm{SU}(2)$, we can decompose the nucleon EM form factors into those of the up and down quarks as follows 25, 26]:

$$
\begin{aligned}
& G_{E, M}^{u}=2 G_{E, M}^{p}+G_{E, M}^{n}, \\
& G_{E, M}^{d}=G_{E, M}^{p}+2 G_{E, M}^{n} .
\end{aligned}
$$

In Fig. 2, we draw the results of the up and down EM form factors in free space (dashed curve) and at normal nuclear matter density (solid curve). In general, the form factors in nuclear matter tend to fall off faster than those in free space. The down EM form factors are shown to be more sensitively affected by the presence of nuclear matter. Interestingly, the in-medium down magnetic form factor starts to decrease less than that in free space till about $Q^{2} \approx 0.3 \mathrm{GeV}^{2}$, and then drops off sharply. We will soon see a similar feature when the transverse charge densities are considered.

The transverse charge densities of quarks inside a nucleon reveal the modification of the nucleon EM structures more prominently. In fact, the usual charge and magnetization densities obtained from the threedimensional Fourier transform of the EM form factors in the Breit framework obscure their physical meaning because of the Lorentz contraction of the nucleon in its moving direction [14, 27. On the other hand, the transverse charge density provides a clear understanding of how the charge of quarks inside a nucleon are distributed. We can extend straightforwardly this to nuclear matter. However, We want to mention a caveat. Since the original Skyrme model produces the underestimated magnetic form factors of the nucleon but explains reasonable $Q^{2}$ dependence, whereas it yields the overestimated electric one of the neutron. It causes a difficulty in describing 

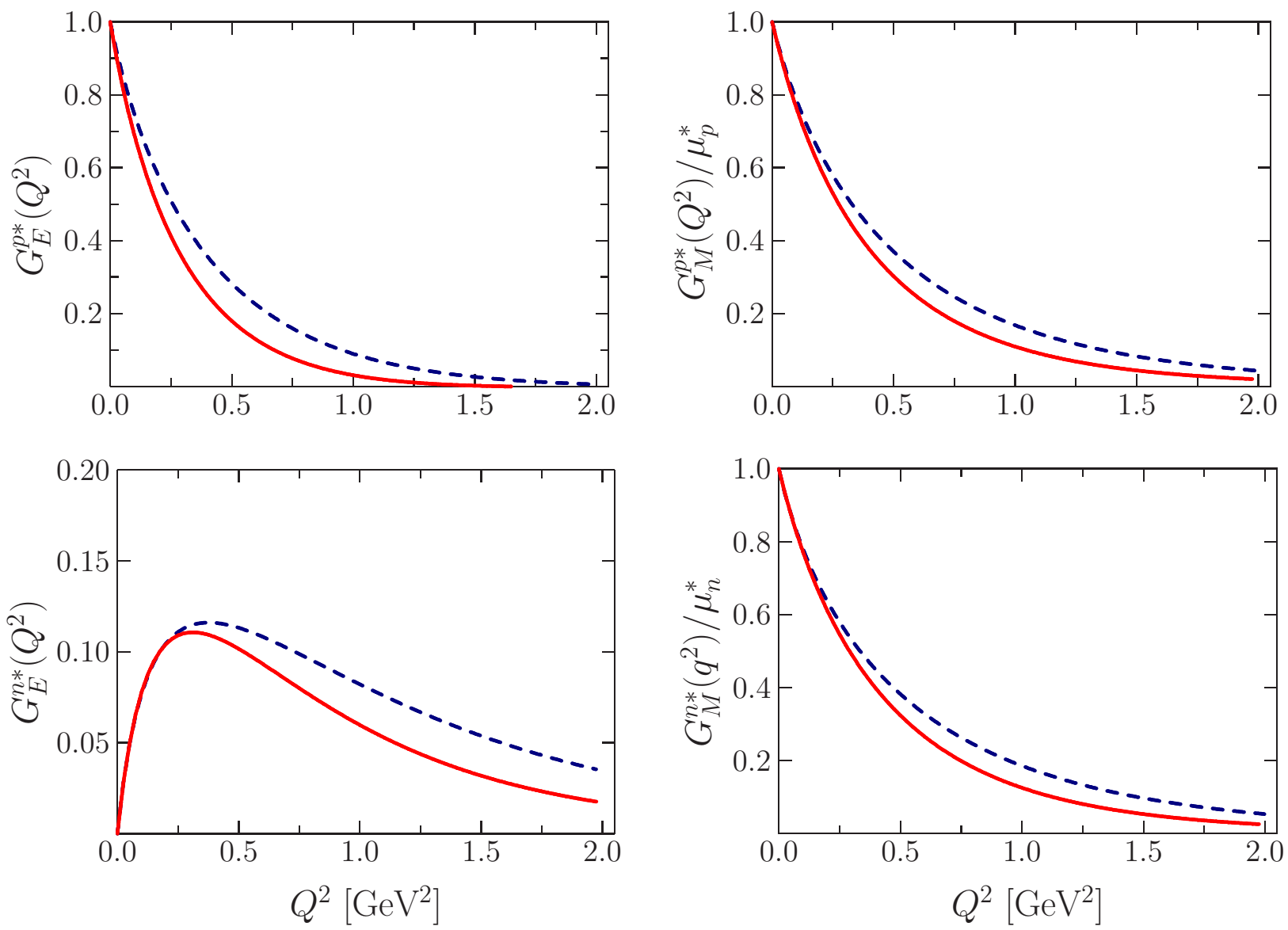

FIG. 1. (Color on line) The electric (left panels) and the normalized magnetic (right panels) form factors of the nucleons as a function of squared momentum transfer $Q^{2}$. The dashed curve corresponds to the free space case, the solid one in nuclear matter with the normal nuclear matter density $\rho_{0}=0.5 m_{\pi}^{3}$.

the transverse charge density inside a neutron that depend on both the neutron electric and magnetic form factors. However, the present aim is at studying how the EM properties of the nucleon undergo changes in nuclear matter, so that we will rather use the normalized nucleon magnetic form factors with the experimental data for the corresponding magnetic moments as shown in Fig. 1 .

When the nucleon is unpolarized, the quark transverse charge density can be expressed as

$$
\rho_{0}^{*}(b)=\int_{0}^{\infty} \frac{Q d Q}{2 \pi} J_{0}(b Q) \frac{G_{E}^{*}\left(Q^{2}\right)+\tau G_{M}^{*}\left(Q^{2}\right)}{1+\tau},
$$

where $\tau=Q^{2} / 4 m_{N}^{2}$ and $J_{0}$ is a Bessel function of order zero. Figure 5 depicts the transverse charge densities inside an unpolarized proton in the upper panel and an unpolarized neutron in the lower panel. In the left and right panels, we show those in free space and in nuclear matter, respectively. The results indicate that the nucleons swell up indeed in nuclear medium. In the center of the proton, the positive charge distribution tends to lessens but it extends in outer directions in nuclear matter. In the case of the neutron, the medium effects are more noticeably observed as shown in the lower-right panel of Fig. 4. Note, however, that the negative charges are centered as in Ref. [16] but are not as deep as that of Ref. 16. The reason lies in the fact that the neutron electric form factor turns out to be overestimated, as mentioned already.

Assuming that the nucleon is polarized in the $x y$ direction which can be expressed by the transverse spin operator of the nucleon $\boldsymbol{S}_{\perp}=\cos \phi_{S} \hat{\boldsymbol{e}}_{x}+\sin \phi_{S} \hat{\boldsymbol{e}}_{y}$, we find that the Pauli form factor contributes to the transverse quark charge densities inside a transversely polarized nucleon as follows:

$$
\begin{aligned}
& \rho_{T}^{*}(\boldsymbol{b})=\rho_{0}^{*}(b)-\sin \left(\phi_{b}-\phi_{S}\right) \\
& \quad \times \int_{0}^{\infty} \frac{Q^{2} d Q}{4 \pi m_{N}} J_{1}(b Q) \frac{-G_{E}^{*}\left(Q^{2}\right)+G_{M}^{*}\left(Q^{2}\right)}{1+\tau}
\end{aligned}
$$

where $\boldsymbol{b}=b\left(\cos \phi_{b} \hat{\boldsymbol{e}}_{x}+\sin \phi_{b} \hat{\boldsymbol{e}}_{y}\right)$ denotes the position vector from the center of the nucleon in the transverse plane. The $J_{0,1}$ are the Bessel functions of order 0 and 1 . The second term in Eq. (14) makes the transverse charge densities distorted from the unpolarized one. We choose the polarization direction of the nucleon along the $x$ axis, i.e. $\phi_{S}=0$.

In Fig. 5, we draw the transverse charge densities in- 

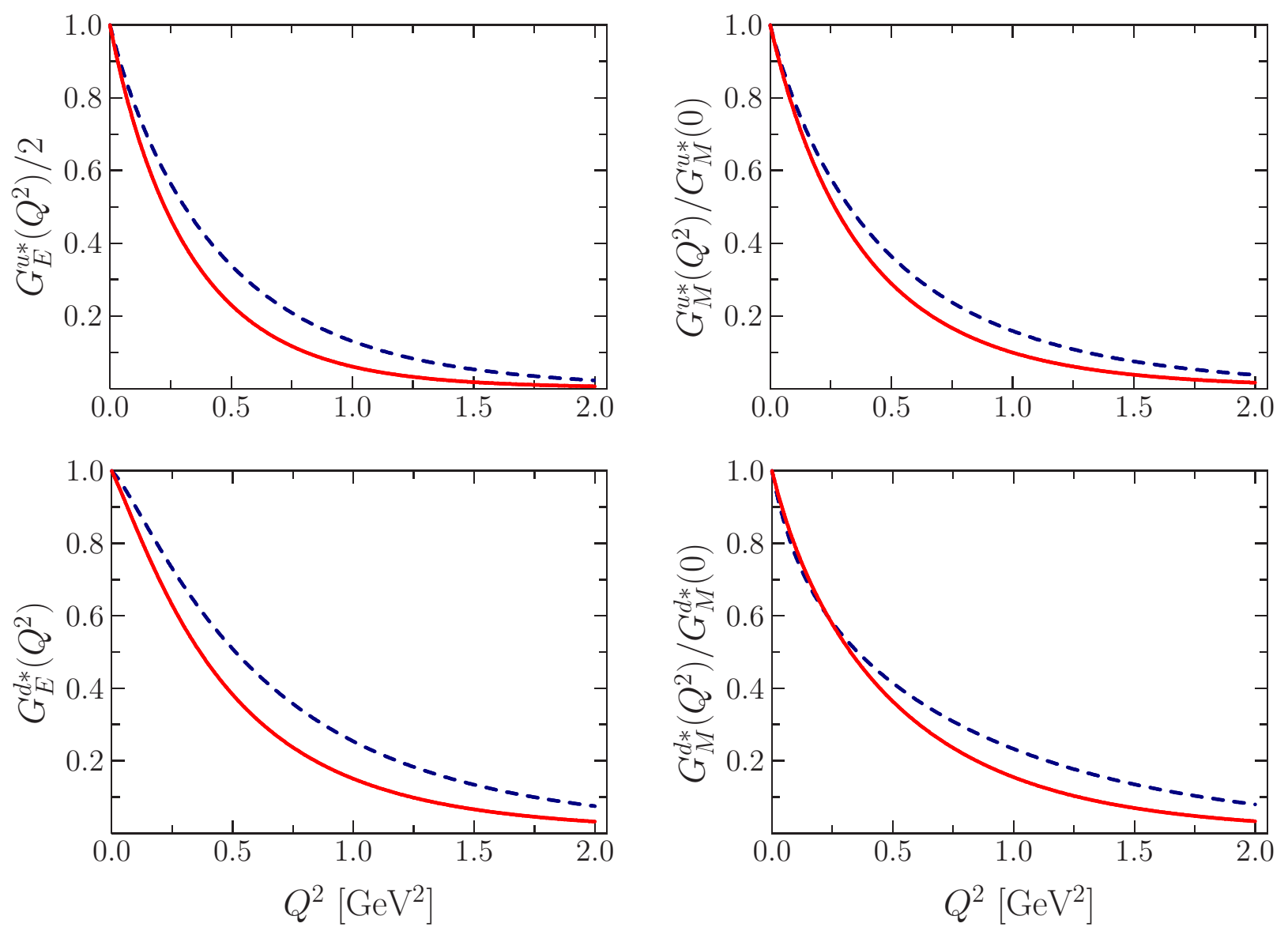

FIG. 2. The electric (left panels) and normalized magnetic (right panels) form factors of the up (upper panels) and down (lower panels) quarks inside a nucleon in nuclear matter as a function of squared momentum transfer $Q^{2}$. Other notations are the same as in Figure 1.

side polarized nucleons. The density inside the polarized nucleon is shown to be distorted in the negative $b_{y}$ direction. As explained already in Refs. [14, 17, the polarization of the nucleon in the $x$ direction induces the electric dipole moment in the negative $y$ direction, which is just the relativistice effect. As a result, the strong distortion arises as shown in Fig. 5. In nuclear matter, the transverse charge density shows the same tendency but seems less obvious. This might be due to the fact that the quark charges decrease in the center of the proton but extend to the outer region. Hence, the influence of the proton polarization on the transverse charge densities is somewhat diminished.

When it comes to the neutron case, the polarization effects are profound: the negative charges are forced to move to the negative $y$, while the positive charges are found in the positive $y$. As a result, the transverse charge densities inside the polarized neutron turn out be very asymmetric. The same is true in nuclear matter. In Fig. 5, the profiles of the transverse charge densities are plotted with $b_{x}$ is fixed to be zero. The effects of nuclear medium are clearly observed. In general, the transverse charge densities in nuclear matter are likely to decrease in the center but extends to the outer directions.

5. The present work aimed at investigating how the electromagnetic properties of the nucleons were modified in nuclear matter within the framework of the in-medium modified Skyrme model. We found that the electromagnetic form factors of the nucleon fell off faster in nuclear matter. The transverse charge densities inside a proton and a neutron indicate that the nucleon in nuclear medium tends to swell up in general. The effects of the proton polarization are somewhat reduced in nuclear matter. Since the positive charges decrease in the center of the proton but extends to outer directions, the distortion of the transverse charge distribution is less noticeable. The same features were found in the neutron case.

The present results are consistent with those of other models: for example, a soliton model with vector mesons 24] and the QMC model calculations [23]. The present investigation may shed light on understanding the medium modification of the nucleon, which will be measured experimentally in near future. 

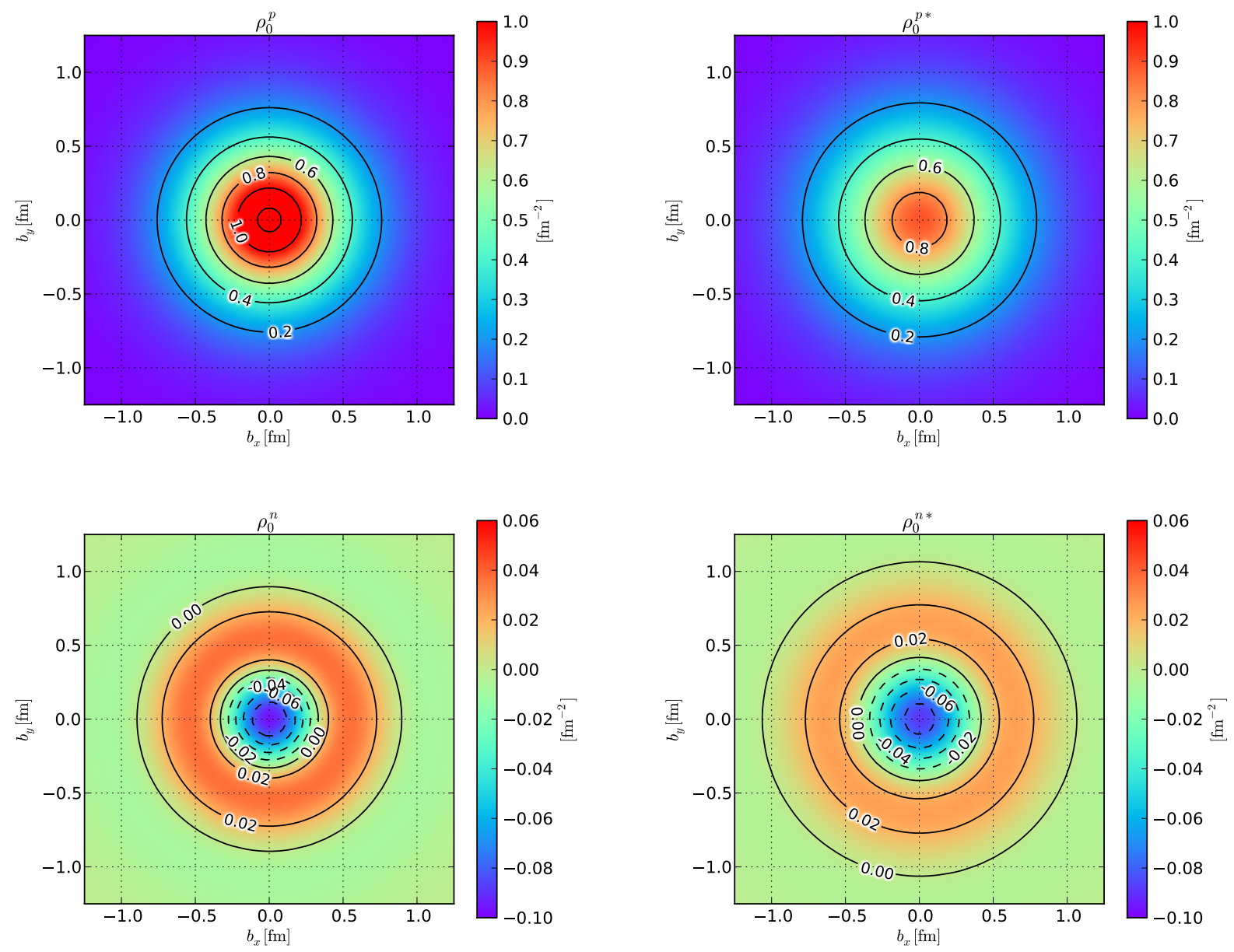

FIG. 3. Quark transverse charge densities inside an unpolarized proton (upper panels) and a neutron (lower panels) in free space (left panels) and at nuclear matter density $\rho_{0}=0.5 m_{\pi}^{3}$ (right panels).

\section{ACKNOWLEDGMENTS}

H.Ch.K is grateful to P. Navratil and R. Woloshyn at TRIUMF for their hospitality during his visit, where the present work was initiated. The authors are thankful to
J.-H. Jung for preparing the figures for the transverse charge densities. The work is supported by Basic Science Research Program through the National Research Foundation of Korea (NRF) funded by the Ministry of Education, Science and Technology Grant Number: 2011-0023478 (U.Y.) and Grant Number: 2012004024 (H.Ch.K.).
[1] M. K. Jones et al. [Jefferson Lab Hall A Collaboration], Phys. Rev. Lett. 84 (2000) 1398 nucl-ex/9910005.

[2] O. Gayou et al. [Jefferson Lab Hall A Collaboration], Phys. Rev. C 64 (2001) 038202.

[3] O. Gayou et al. [Jefferson Lab Hall A Collaboration], Phys. Rev. Lett. 88 (2002) 092301 nucl-ex/0111010.

[4] V. Punjabi et al. [Jefferson Lab Hall A Collaboration], Phys. Rev. C 71 (2005) 055202 [Erratum-ibid. C 71 (2005) 069902] nucl-ex/0501018.

[5] A. J. R. Puckett, E. J. Brash, M. K. Jones, W. Luo, M. Meziane, L. Pentchev, C. F. Perdrisat and V. Pun- jabi et al., Phys. Rev. Lett. 104 (2010) 242301 arXiv:1005.3419 [nucl-ex]].

[6] J. C. Bernauer et al. [A1 Collaboration], Phys. Rev. Lett. 105 (2010) 242001 arXiv:1007.5076 [nucl-ex]].

[7] G. Ron et al. [Jefferson Lab Hall A Collaboration], Phys. Rev. C 84 (2011) 055204 arXiv:1103.5784 [nucl-ex]].

[8] X. Zhan, K. Allada, D. S. Armstrong, J. Arrington, W. Bertozzi, W. Boeglin, J. -P. Chen and K. Chirapatpimol et al., Phys. Lett. B 705 (2011) 59 arXiv:1102.0318 [nucl-ex]]. 

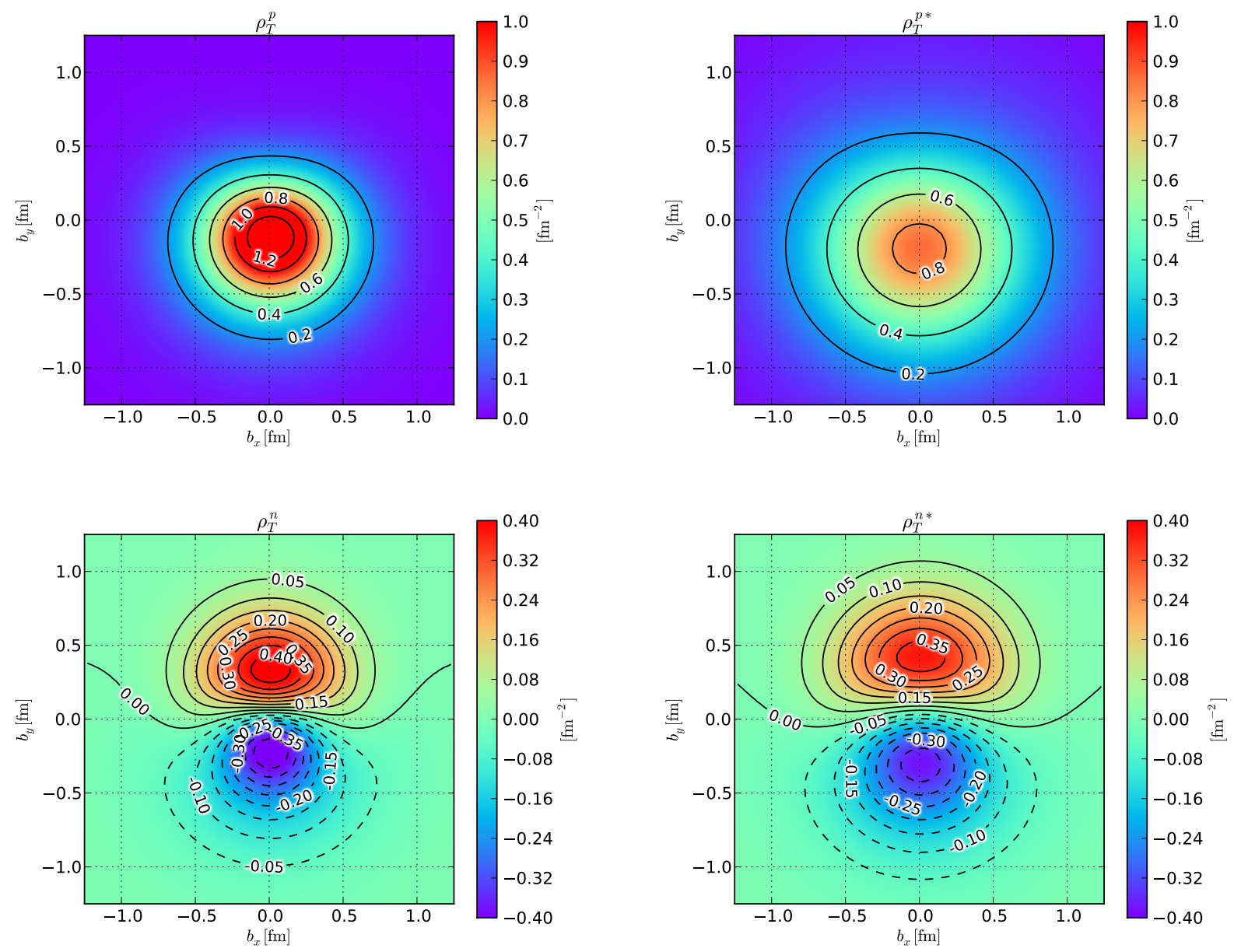

FIG. 4. Transverse charge densities of the proton (upper panels) and neutron (lower panels) in free space (left panels) and in nuclear matter with the density $\rho_{0}=0.5 m_{\pi}^{3}$ (right panels).

[9] C. E. Hyde-Wright and K. de Jager, Ann. Rev. Nucl. Part. Sci. 54 (2004) 217 nucl-ex/0507001.

[10] J. Arrington, C. D. Roberts and J. M. Zanotti, J. Phys. G 34 (2007) S23 nucl-th/0611050.

[11] C. F. Perdrisat, V. Punjabi and M. Vanderhaeghen, Prog. Part. Nucl. Phys. 59 (2007) 694 hep-ph/0612014.

[12] G. D. Cates, C. W. de Jager, S. Riordan and B. Wojtsekhowski, Phys. Rev. Lett. 106, 252003 (2011) arXiv:1103.1808 [nucl-ex]].

[13] I. A. Qattan and J. Arrington, Phys. Rev. C 86, 065210 (2012) arXiv:1209.0683 [nucl-ex]].

[14] M. Burkardt, Int. J. Mod. Phys. A 18 (2003) 173 hep$\mathrm{ph} / 0207047$.

[15] M. Burkardt, Phys. Rev. D 62 (2000) 071503 [Erratumibid. D 66 (2002) 119903] hep-ph/0005108.

[16] G. A. Miller, Phys. Rev. Lett. 99 (2007) 112001 arXiv:0705.2409 [nucl-th]].

[17] C. E. Carlson and M. Vanderhaeghen, Phys. Rev. Lett. 100 (2008) 032004 arXiv:0710.0835 [hep-ph]].
[18] A. Airapetian et al. [HERMES Collaboration], Phys. Rev. C 81 (2010) 035202 arXiv:0911.0091 [hep-ex]].

[19] A. Rakhimov, M. M. Musakhanov, F. C. Khanna and U. T. Yakhshiev, Phys. Rev. C 58 (1998) 1738 nuclth/9609049.

[20] U. Yakhshiev and H.-Ch. Kim, Phys. Rev. C 83 (2011) 038203 arXiv:1009.2909 [hep-ph]].

[21] H. -Ch. Kim, P. Schweitzer, and U. Yakhshiev, Phys. Lett. B 718 (2012) 625 arXiv:1205.5228 [hep-ph]].

[22] T. Ericson and W. Weise, Pions and Nuclei (Clarendon, Oxford, 1988).

[23] D. H. Lu, A. W. Thomas, K. Tsushima, A. G. Williams and K. Saito, Phys. Lett. B 417 (1998) 217.

[24] U. G. Meissner, Phys. Rev. Lett. 62 (1989) 1013.

[25] G. A. Miller, B. M. K. Nefkens, I. Slaus, Phys. Rept. 194 (1990) 1.

[26] D. H. Beck, R. D. McKeown, Ann. Rev. Nucl. Part. Sci. 51 (2001) 189 hep-ph/0102334.

[27] J. J. Kelly, Phys. Rev. C 66 (2002) 065203 hep$\mathrm{ph} / 0204239$. 

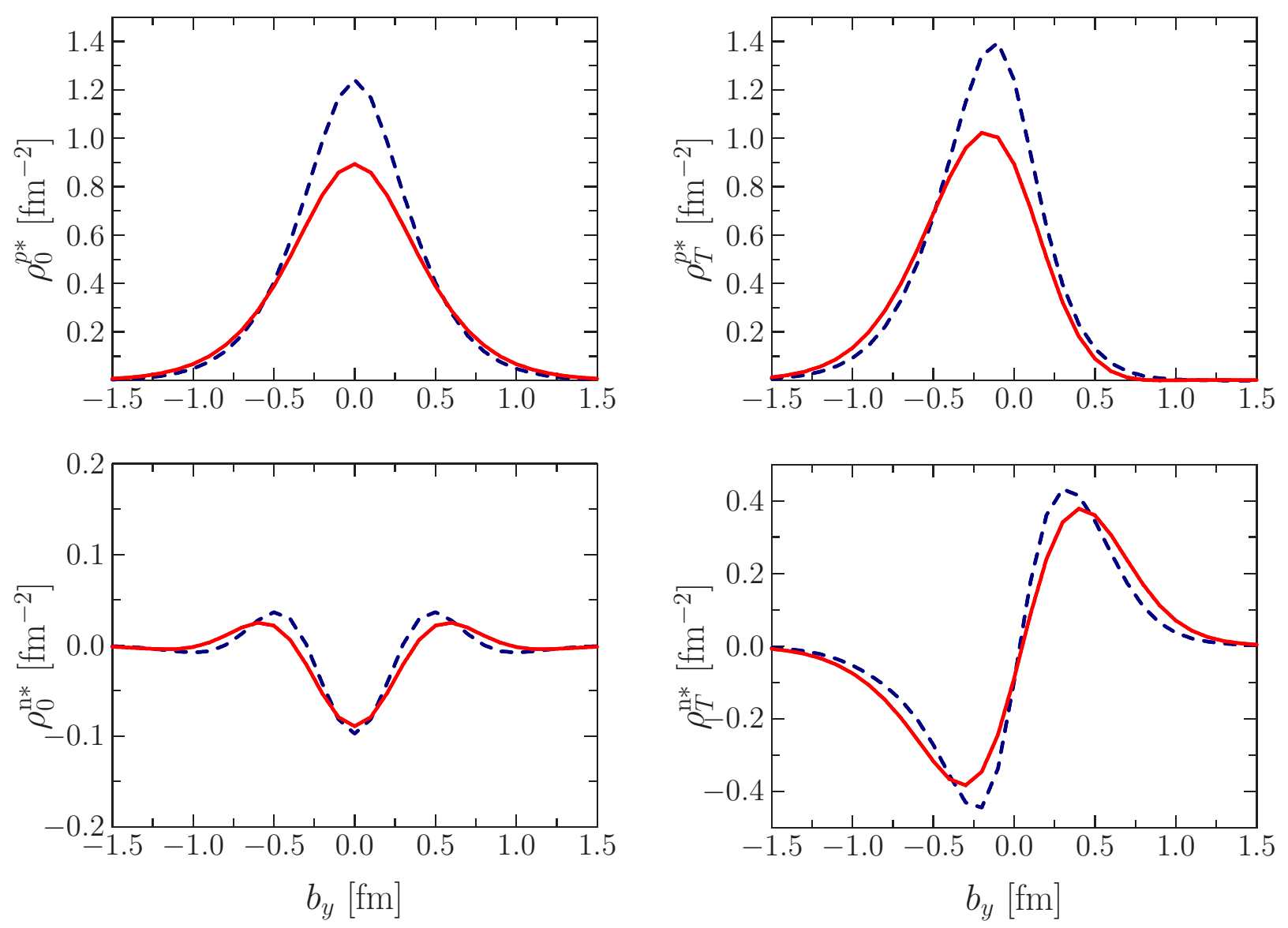

FIG. 5. Transverse charge densities inside unpolarized and polarized protons with $b_{x}$ fixed to be zero are plotted in the upperleft and the upper-right panels, respectively. In the lower panels, those inside neutrons are presented with the same notation as the proton case. The dashed curves draw the results in free space and the solid ones represent those in nuclear matter at normal nuclear matter density $\rho_{0}=0.5 \mathrm{~m}_{\pi}^{3}$. 